\title{
Soziologie der Unternehmerfamilie
}

\author{
Patrick Kahle
}

Online publiziert: 30. März 2020

(c) Der/die Autor(en) 2020

Kleve, Heiko, und Tobias Köllner (Hrsg.): Soziologie der Unternehmerfamilie. Grundlagen, Entwicklungslinien, Perspektiven. Wiesbaden: Springer VS 2019. 299 Seiten. ISBN: 978-3-658-22387-8. Preis: $€ 44,99$.

Innerhalb der Soziologie ist die Unternehmerfamilie ein bisher wenig beachtetes Thema. Und dies, obwohl Familienunternehmen je nach Studie zwischen 71 und $96 \%$ aller Unternehmen in Deutschland ausmachen (S. 115). Der Sammelband stellt sich der Aufgabe, einen Beitrag zur Zusammenführung verschiedener Befunde und Ansätze der Analyse von Familienunternehmen und Unternehmerfamilien zu leisten (S. 2).

Nebst Einführung besteht der Band aus drei Teilen mit unterschiedlicher Beitragszahl. In der Einführung durch die beiden Herausgeber wird die Unternehmerfamilie als ,eine Verwandtschaftsgruppe“ definiert, ,die ganz oder teilweise an einem Unternehmen beteiligt ist und dabei einen entscheidenden Einfluss auf das Unternehmen nimmt, und zwar entweder durch operative Mitarbeit oder als aktive Eigentümerbzw. Gesellschafterfamilie“ (S. 2). Im Anschluss werden der Aufbau und die Beiträge des Bands vorgestellt.

Der erste Teil, „Grundlagen“, beginnt mit einem Beitrag von Hans Bertram, der die familiäre Produktion von Humankapital seit dem 19. Jahrhundert darstellt. Dabei wird einerseits darauf aufmerksam gemacht, dass Erwerbstätigkeit von Frauen stets häufiger war und ist als in den gesellschaftlichen Vorstellungen angenommen, und andererseits die Kindheit keine größere Strukturierung erfahren kann. Angesichts der Unterbrechungen und fehlenden Aufstiegschancen in weiblichen Erwerbsbiografien

P. Kahle $(\bowtie)$

Institut für Sozialwissenschaften, Universität Hildesheim

Universitätsplatz 1, 31141 Hildesheim, Deutschland

E-Mail: kahlep@uni-hildesheim.de 
wird am Ende des Beitrags im Stile einer Expertise für ein Modell institutionalisierter zweiter Bildungswege etwa ab dem 45. Lebensjahr plädiert. Désirée Waterstradt beleuchtet vor dem Hintergrund der Prozesssoziologie von Elias die Entwicklung des Verhältnisses von Familien und Haushalt seit der europäischen Vorgeschichte. Dabei nimmt sie zunächst die Entmythisierung romantischer Familienvorstellungen (Stammfamilie und Ganzes Haus, S. 62) und der eindeutigen Grenzziehung zwischen Familie und Wirtschaften vor. Anschließend geht sie auf Figurationen - über dem, oikos, domus, familia, Haus - bis in die Gegenwart - Familie und Unternehmen (S. 82 ff.) - ein, die sich aus der Wechselwirkung von Produktionseinheiten, Eheund Geschlechterverhältnissen sowie dem jeweiligen Familienbegriff ergeben. Stefan Kühl nimmt in seinem Beitrag dezidiert eine systemtheoretische Perspektive ein, die auch bei der Mehrheit der folgenden Beiträge vorherrscht. Seine These ist, dass sich neben der funktionalen Differenzierung und der Ebenendifferenzierung zwischen Interaktionen, Organisationen und Gesellschaft auf der Ebene der Organisationen ,auch andere soziale Zusammenhänge wie Gruppen, Bewegungen und Familien [ausdifferenzieren]“ (S. 99). Auf dieser Ebene seien diese sozialen Zusammenhänge zwar zu trennen, ,können [...] aber auch ineinander verschachtelt [und verwoben] werden“ (S. 110f.). Isabell Stamm, Fabian Bernhard und Nicole Hameister thematisieren in ihrem Beitrag zunächst die Datengrundlage zu Unternehmerfamilien in quantitativen Studien. Daran schließt ein Vergleich zwischen einer Stichprobe aus dem pairfam-Datensatz und einer mittels Propensity Score Matching methodisch anspruchsvoll konstruierten Vergleichsgruppe an (jeweils $n=337$ Individuen). Befund ihres Vergleichs ist, dass Mitglieder von Familienunternehmen über eine höhere und ungeregelte Wochenarbeitszeit verfügen (S. 136).

Zu Beginn des 2. Teils, „Entwicklungslinien“, gehen Rudolf Wimmer und Fritz B. Simon aus systemtheoretischer Perspektive dem Strukturwandel der Familie beim Übergang zur Moderne nach. Das konstituierende Dritte einer Familie ist klassisch das Kind, in Unternehmerfamilien gesellt sich das Unternehmen hinzu. In allen Interaktionen der Unternehmerfamilie sind beide Belange stets präsent und setzen entweder ein wortloses Verstehen voraus oder führen unweigerlich zu Konflikten (S. 162-166). Gabriela Leiß geht - methodisch an der Grounded Theory von Strauß und Corbin orientiert - Aushandlungsmustern der Nachfolge in Familienunternehmen in der reflexiven Moderne nach. Vier dieser Muster illustriert sie anhand von Fallbeispielen. So unterscheidet sie zwischen ,,autoritärer Sicherung“ (S. 176), ,ambivalente[r] Verstrickung“ (S. 177), ,unabhängige[r] Erneuerung“ (S. 179) und ,[k]o-evolutionäre[r] Entwicklung“ (S. 180), wobei Leiß für Letztere als erfolgversprechendstes Muster plädiert. Daniela Jäkel-Wurzer thematisiert in ihrem Beitrag die Beteiligung von Frauen als Eigentümerinnen und weibliche Nachfolge vor dem Hintergrund sich wandelnder Familienvorstellungen. Unternehmerfamilien als Familien eigener Art können ,die Autonomieimpulse ihrer Nachkommen nicht uneingeschränkt unterstützen“ (S. 192). Während aber historisch weibliche Nachfolge ausgeschlossen war, machen Exit-Optionen der Söhne und Kompetenzentscheidungen weibliche Nachfolge wahrscheinlicher. Dennoch sind Frauen sozial stärker an die Mutterrolle gebunden als Männer an die Vaterrolle, weswegen Rollenambiguitäten und erschwerte Vereinbarkeit Nachfolgerinnen vor besondere Herausforderungen stellen. Im Beitrag von Elke Schröder werden Sozialisationsprozesse in Unterneh- 
merfamilien anhand empirischen Materials illustriert. Er dient zur „Anregung für Unternehmenseltern, wie sie die soziale Umwelt ihrer Kinder möglichst förderlich gestalten und zur Nachhaltigkeit des Familienunternehmens beitragen können“ (S. 206).

Der dritte Teil, „Perspektiven“, enthält zunächst zwei Beiträge von Tom A. Rüsen, Arist von Schlippe und Heiko Kleve. In ihrem ersten Beitrag thematisieren sie die dynastische Großfamilie als spezifischen Typus von Unternehmerfamilien und geben dieser einen Leitfaden oder Leitfragen, um sich zu organisieren, an die Hand. In ihrem zweiten Beitrag thematisieren sie Unternehmerfamilien als ,,verdreifachte" Familien, die sowohl Familie, Organisation als auch Netzwerk sind. Auch dieser Beitrag kann als Handreichung gelesen werden, Unternehmerfamilien - vor allem weit verzweigte mit ihren spezifischen Kommunikations- und Organisationsproblemen (wie z.B. Multilingualität und -generationalität) - zu organisieren. Von Schlippe und Groth spüren in ihrem Beitrag theoretisch anspruchsvoll einer systemtheoretischen Erweiterung des Drei-Kreis-Denkens nach. Personen in Unternehmerfamilien sind ständig mit den Imperativen konfrontiert, sich „familien-konform“ und „unternehmerfamilien-konform“ zu verhalten, welche nicht widerspruchsfrei sind. Dies sei vor allem in der Beratung von Unternehmerfamilien eine hilfreiche Erkenntnis. Kleve geht im abschließenden Beitrag der Frage nach, ,ob wir Unternehmerfamilien als einen eigenständigen Systemtyp“ im Sinne der Luhmann'schen Systemtheorie „bewerten können“ (S. 282). Als Funktion identifiziert er dabei, „die Einführung familiärer Langfristperspektiven“ (S. 295) in ein zeitlich beschleunigtes Wirtschaftssystem. Der Code ergebe sich aus dem Bestehen am Markt.

In seiner Form bietet der Sammelband sowohl Beiträge aus der grundbegrifflichen Arbeit, aus Forschungsprojekten, die weitere Desiderata explizieren, als auch aus Beratungskontexten. Er stellt damit einen profunden Einführungsband für Mitglieder von Unternehmerfamilien und Personen dar, die einen Einstieg in dieses Forschungsfeld suchen. Blickt man auf die skizzierten Beiträge, so tritt eine Vielzahl nicht nur thematischer, sondern auch theoretischer Überschneidungen auf. So hätte neben den systemtheoretischen Aufsätzen auch einer mit Provenienz aus der Soziologie der Konventionen, die sich in den letzten Jahren auch im deutschsprachigen Raum der (Wirtschafts-)Organisationsforschung vermehrt zugewandt hat, den Band bereichert. Thematisch werden im Band die öffentliche Wahrnehmung und gesellschaftliche Verantwortung von Unternehmerfamilien wiederholt angeschnitten, sodass ein Beitrag explizit zu reputationstheoretischen Fragen die vorhandenen gut ergänzt hätte. Inhaltliche Redundanzen hätten durch eine leicht veränderte Komposition der Beiträge vermieden werden können - etwa indem einzelne Beiträge einen stärker kommentierenden Charakter gegenüber anderen (z. B. Schlippe und Groth zu Kühl oder Jäkel-Wurzer zu Waterstradt) eingenommen hätten. Ein weiterer Band mit - zielgruppenspezifischem - Handbuchcharakter wird sicherlich auch auf geneigte Leserschaft stoßen.

Funding Open Access funding provided by Projekt DEAL.

Open Access Dieser Artikel wird unter der Creative Commons Namensnennung 4.0 International Lizenz veröffentlicht, welche die Nutzung, Vervielfältigung, Bearbeitung, Verbreitung und Wiedergabe in jeglichem Medium und Format erlaubt, sofern Sie den/die ursprünglichen Autor(en) und die Quelle ord- 
nungsgemäß nennen, einen Link zur Creative Commons Lizenz beifügen und angeben, ob Änderungen vorgenommen wurden.

Die in diesem Artikel enthaltenen Bilder und sonstiges Drittmaterial unterliegen ebenfalls der genannten Creative Commons Lizenz, sofern sich aus der Abbildungslegende nichts anderes ergibt. Sofern das betreffende Material nicht unter der genannten Creative Commons Lizenz steht und die betreffende Handlung nicht nach gesetzlichen Vorschriften erlaubt ist, ist für die oben aufgeführten Weiterverwendungen des Materials die Einwilligung des jeweiligen Rechteinhabers einzuholen.

Weitere Details zur Lizenz entnehmen Sie bitte der Lizenzinformation auf http://creativecommons.org/ licenses/by/4.0/deed.de.

Patrick Kahle M.A., Mitarbeiter am Institut für Sozialwissenschaften der Universität Hildesheim. Forschungsschwerpunkte: Kollektive Identität, Solidarität, Diskurstheorie und -analyse, Text Mining. Aktuelle Publikationen: Middelschulte, Henning, und Kahle, Patrick: Ökonomisierung der sozialwissenschaftlichen Bildung? Anwendung eines konventionensoziologischen Diktionärs zur massentextanalytischen Untersuchung einer bildungspolitischen Debatte. In: Imdorf, Christian; Leemann, Regula und Gonon, Philipp (Hrsg.): Bildung und Konventionen. Die ,Économie des Conventions' in der Bildungsforschung. Wiesbaden: Springer VS 2019, S. 255-280. 